\title{
Occurrence of Aflatoxigenic Aspergillus Species in Peanut Varieties in Busia and Kisii Central Districts, Kenya
}

\author{
Menza C. Nelson, Muturi W. Margaret* \\ Department of Medical Laboratory Sciences, Kenyatta University, Nairobi, Kenya \\ Email: *muturi.margaret@ku.ac.ke, menzanelson2010@gmail.com, menza.nelson@ku.ac.ke
}

How to cite this paper: Nelson, M.C. and Margaret, M.W. (2018) Occurrence of Aflatoxigenic Aspergillus Species in Peanut Varieties in Busia and Kisii Central Districts, Kenya. Open Journal of Medical Microbiology, 8, 98-108.

https://doi.org/10.4236/ojmm.2018.84009

Received: August 21, 2018

Accepted: November 5, 2018

Published: November 8, 2018

Copyright $\odot 2018$ by authors and Scientific Research Publishing Inc. This work is licensed under the Creative Commons Attribution International License (CC BY 4.0).

http://creativecommons.org/licenses/by/4.0/

\section{(c) (i) Open Access}

\begin{abstract}
Recent studies have shown that peanuts in Kenya are highly contaminated with aflatoxins, however, information gaps exist on the characterization of the Aspergillus species that produce aflatoxins. Therefore, this gap necessitated the determination of the Aspergillus species producing aflatoxins in peanuts from the main growing districts of Busia and Kisii central. One hundred and two (102) peanuts samples were collected from farmers' in each district and Aspergillus species were isolated using the dilution plate technique on modified Rose Bengal Agar. Phenotypical characterization of the identified Aspergillus flavus isolates from the samples was determined using the procedure of Mellon and Cotty. This study identified 5 Aspergillus species as contaminants in peanut analyzed. They were Aspergillus flavus L-strain, Aspergillus flavus S-strain, Aspergillus parasiticus, Aspergillus niger and Aspergillus tamari. Overall, the occurrence of Aspergillus flavus $\mathrm{L}$-strain and $A$. flavus $\mathrm{S}$-strain was significantly higher than other species identified $(\mathrm{H}=15.55, \mathrm{df}=4, \mathrm{P}=0.004)$ in peanuts from the two districts. Aspergillus flavus L-strain was the most common isolate (58.8\%) in peanut from Busia district while $A$. flavus $\mathrm{S}$-strain was the most common strain $(60.2 \%)$ in peanuts from Kisii central district. However, A. flavus S-strain was the most dominant species $(\mathrm{F}=3.15, \mathrm{df}=25, \mathrm{P}=0.031)$ with an overall mean occurrence of $45.1 \%$. The confirmation of occurrence of other species that produce toxins such as $A$. niger and $A$. tamarii which also produces cyclopiazonic acid, points to the need of screening peanuts for other carcinogenic mycotoxins.
\end{abstract}

\section{Keywords}

Aflatoxins, Peanuts, Aspergillus Species 


\section{Introduction}

Aflatoxins are highly carcinogenic mycotoxins that are produced by Aspergillus species, specifically Aspergillus flavus and Aspergillus parasiticus. Aflatoxins contamination of foods, including peanuts, is a major hazard to human health and has been associated with liver failure, stunted growth in children, hepatocellular carcinoma (HCC) and death [1]. In Kenya, a high incidence rate of liver cancer was reported in the year 2008. It has been reported that in every 100,000 people suffering from liver cancer, $8.5 \%$ were males while $4.9 \%$ were females [2] worldwide. Aflatoxins are produced by Aspergillus species in food crops such as peanuts when they are poorly dried and stored [3].

Kenya has repeatedly experienced epidemics of acute aflatoxicosis especially in the Eastern province in the years 2001, 2004, 2005 and 2006 [4]. The largest outbreak due to maize aflatoxin poisoning was reported in the year, 2004 where 125 people died out of the 317 reported cases [5]. Studies conducted in other developing countries established a relationship between levels of aflatoxin and Aspergillus contamination. While both $A$. flavus and $A$. parasiticus can produce aflatoxin B toxins, $A$. parasiticus exclusively produces the G1 and G2 aflatoxins that are associated with liver toxicity and carcinogenicity [3]. Recent studies have shown that peanuts in Kenya, particularly those produced in Busia and Kisii central districts, are highly contaminated with aflatoxins [6], but information gaps exist on the distribution of the producing Aspergillus species. Further, few studies have been done to characterize the fungi that produce the different types of aflatoxins. This gap necessitated the determination of the Aspergillus species producing aflatoxins in peanuts from the main peanut producing districts of $\mathrm{Bu}$ sia and Kisii central districts in western Kenya.

\section{Materials and Methods}

\subsection{Study Areas}

The study was conducted in two districts in western Kenya namely Busia and Kisii central. These are the main peanuts producing districts in the region and have several peanuts processors [7].

\subsection{Study Design}

A cross-sectional study was adopted among the peanuts farmers in Busia and Kisii central districts.

\subsection{Study Population}

The study comprised of peanuts producers in Busia and Kisii central districts.

\subsection{Sampling Technique}

Peanuts farmers' households were purposively sampled from the divisions in the two districts. In Busia, farmers in the leading groundnut producing divisions identified as Butula, Matayos, Funyula and Budalangi were purposively selected. 
In Kisii central, farmers in the four leading peanut producing divisions; Keumbu, Masimba, Suneka and Mosocho were also purposively sampled. Within the divisions, every fourth household of peanuts farmers' was sampled. The sampling interval was obtained based on the approximate peanuts farmers' population of 408 in the study areas [8] divided by the sample size (102).

\subsection{Study Approval, Ethical Consideration and Informed Consent}

Ethical approval was obtained from Kenyatta University Ethics Review Committee. The study objectives were explained to the peanut farmers and they were allowed to ask questions. After giving consent, the farmers who were willing to participate signed a consent form and peanuts samples were collected.

\subsection{Laboratory Analysis}

\subsubsection{Sample Collection}

A total of 102 peanut samples from each peanut farmer's household in each study district were collected using the procedure of Whitaker, (2006) [9]. Each of the $0.5 \mathrm{~kg}$ samples of unsorted peanuts was put in clean polyethylene bags, sealed, labeled and transported in cool boxes to Bora Limited Laboratory, Nairobi and University of Nairobi, Department of Food Science, Nutrition and Technology. They were stored at $4^{\circ} \mathrm{C}$ once received until the time of analysis for incidence, types and levels of aflatoxins in peanuts.

\subsubsection{Aspergillus Species Culture and Identification}

Twenty (20) grams of each peanut sample was grounded using a dry mill kitchen grinder (Kanchan multipurpose Kitchen machine, Kanchan International Limited Mumbai, India) and mixed thoroughly by shaking. Aspergillus species were isolated from the peanut samples by using the dilution plate technique on modified Rose Bengal agar using the procedure of Probst et al., 2007 [10].

The colonies of Aspergillus species were sub-cultured on $9 \mathrm{~cm}$ diameter petridishes containing $20 \mathrm{ml}$ of Malt Extract Agar (MEA) and Czapek-Dox agar $(\mathrm{CZ})$, and then incubated for 7 days in the dark at $25^{\circ} \mathrm{C}$. They were subsequentially examined for coloured colonies, presence and size of sclerotia, head seriation and conidial morphology. All isolates were also cultured on Aspergillus flavus parasiticus agar (AFPA) for 3 - 5 days at $25^{\circ} \mathrm{C}$ in the dark to confirm group identification by colony reverse colour. All isolates were subsequently cultured on $\mathrm{CZ}$ agar at $42^{\circ} \mathrm{C}$ and colony diameters measured after 7 days of incubation. Identification of species isolates was done according to Klich, (2002) [11], and by comparison with reference strains obtained from Dr. Bruce Horn (USDA National Peanut Research Lab, Dawson, Georgia, United States of America).

\section{Aspergillus flavus strains characterization}

Phenotypical characterization of the identified Aspergillus flavus isolates from the peanuts samples was determined using the procedure of Mellon and Cotty, (2004) [12]. The isolates were then classified on the basis of colony characteristics and conidial morphology at $400 \times$ magnification using a high resolution mi- 
croscope. Colony radius was measured in millimeter $(\mathrm{mm})$ and the colony color of isolates determined using Methuen color book [13]. Isolates with abundant small sclerotia (average diameter $<400 \mathrm{~mm}$ ) were classified as strain S of $\mathrm{A}$. flavus. Isolates with smooth conidia and large sclerotia (average diameter over 400 $\mathrm{mm}$ ) were classified as the $\mathrm{L}$ strain of $A$. flavus [14].

\section{Results}

\subsection{Peanuts Varieties from Busia and Kisii Central Districts}

A total of 204 peanut samples of different varieties were collected in the two districts. In Busia district, the 102 peanut samples were of four different varieties; Valencia red, Uganda local, Homabay local and Local red. The 102 peanut samples from Kisii central district were of three different varieties; Valencia red, Uganda local and Homabay local. In both districts, Valencia red variety had the most number of the samples, 59 and 89 from Busia and Kisii central districts respectively which were significantly different from the other varieties $\left(\chi^{2}=12.00\right.$, $\mathrm{df}=9, \mathrm{P}=0.02)$. There were more samples of Uganda local red (21) and Homabay local (20) varieties from Busia district compared to those from Kisii central district. Local red variety had only 2 samples in Busia and none in Kisii central (Table 1).

\subsection{Occurrence of Aspergillus Species in Peanuts}

Five (5) Aspergillus species were identified as contaminants in peanuts analyzed in this study. They were Aspergillus flavus L-strain, Aspergillus flavus S-strain, Aspergillus parasiticus, Aspergillus niger and Aspergillus tamarii. Overall, the occurrence of Aspergillus flavus L-strain and A. flavus S-strain were significantly higher than other species identified $(\mathrm{H}=15.55, \mathrm{df}=4, \mathrm{P}=0.004)$ in peanut samples from the two districts. However, A. flavus S-strain was the most dominant species identified in the study with a mean occurrence of $45.1 \%$ (Table 2). Aspergillus flavus L-strain was the most common isolate (58.8\%) in Busia district while A. flavus S-strain was the most common strain (60.2\%) in Kisii central district (Table 2).

Aspergillus parasiticus was the third most common isolate in samples from

Table 1. Peanut varieties from Busia and Kisii central districts.

\begin{tabular}{cccc}
\hline & \multicolumn{3}{c}{ Number of peanut samples collected } \\
\hline Peanut variety & Busia & Kisii central & Total \\
\hline Valencia red $^{\mathrm{a}}$ & 59 & 89 & 148 \\
Uganda local red & 21 & 5 & 26 \\
Homa Bay local & 20 & 8 & 28 \\
Local red & 2 & 0 & 2 \\
Total & 102 & 102 & 204 \\
\hline
\end{tabular}

$\mathbf{a}=$ peanut variety with a significantly higher number of samples in the study. 
Table 2. Occurrence of Aspergillus species strains in peanut samples.

\begin{tabular}{ccccc}
\hline & \multicolumn{2}{c}{ Busia } & \multicolumn{2}{c}{ Kisii central } \\
\hline Aspergillus species isolated & & $\%$ & & $\%$ \\
\hline A. flavus L-strain & 60 & 58.8 & 22 & 21.8 \\
A. flavus S-strain & 30 & 29.4 & 62 & 60.2 \\
A. parasiticus & 7 & 6.9 & 12 & 12.0 \\
A. niger & 2 & 2.0 & 4 & 4.0 \\
A. tamarii & 0 & 0.0 & 2 & 2.0 \\
Negative for Aspergillus species & 3 & 2.9 & 0 & 0.0 \\
\hline
\end{tabular}

both districts at $12 \%$ and $6.9 \%$ for Kisii central and Busia districts respectively. Other species including Aspergillus niger was isolated at $2 \%$ and $4 \%$ in peanut samples from Busia and Kisii central districts respectively. Aspergillus tamarii was the least occurring species at $2 \%$ in peanuts from Kisii central district (Table 3). The mean occurrence for Aspergillus tamarii was 1\% in both districts (Table 3). Only, $2.9 \%$ of peanut collected from Busia district were negative for Aspergillus species contamination while all peanut from Kisii central district were contaminated with at least one aflatoxin producing species (Table 3 ).

\section{Aspergillus Species in the Different Varieties of Peanuts}

All the samples were contaminated with at least one or more of A. flavus L-strain, A. flavus S-strain, A. parasiticus, Aspergillus niger and A. tamari species. Overall, the result showed that the incidence of Aspergillus flavus S-strain was significantly higher than other Aspergillus species identified $(\mathrm{F}=3.15, \mathrm{df}=$ $25, \mathrm{P}=0.031$ ).

Aspergillus flavus L-strain was the most highly detected strain (60.6\%) in all the peanut varieties from Busia district compared to the other Aspergillus species isolates $(\mathrm{H}=10.03, \mathrm{df}=3, \mathrm{P}=0.018)$. It was mostly found in Homabay local variety peanut samples with $33.3 \%$ occurrence (Table 4). Aspergillus flavus S-strain was the most abundant species in peanut samples of the Homabay local in Busia district at an incidence of $40 \%$. Aspergillus parasiticus was also found to be contaminating all the peanut varieties from the study district but was isolated highly in peanuts of local red, Valencia red and Uganda local red varieties at an incidence of $28.6 \%$. Aspergillus niger was only detected in all the peanuts of Local red variety while $A$. tamarii was not detected in any peanut varieties from Busia district (Table 4).

All the strains of Aspergillus except $A$. tamarii were isolated in all the peanut varieties from Kisiicentral district. However, Aspergillus flavus $S$-strain had higher occurrence at $60.8 \%$ compared to other species identified in peanuts from the district $(\mathrm{H}=12.28, \mathrm{df}=4, \mathrm{P}=0.015)$. Aspergillus flavus $\mathrm{S}$-strain was highly detected in samples of Valencia red variety with incidence of $79 \%$ compared to Aspergillus flavus L-strain at $54.6 \%$. Aspergillus parasiticus species was found at an incidence of $41.7 \%$ in Homabay local variety samples while Aspergillus tamarii 
Table 3. Mean occurrence of different Aspergillus species in peanuts from the two districts.

\begin{tabular}{ccc}
\hline Aspergillus species isolated & & $\%$ \\
\hline A. flavus L-strain & 82 & 40.2 \\
A. flavus S-strain & 92 & 45.1 \\
A. parasiticus & 19 & 9.3 \\
A. niger & 6 & 2.9 \\
A. tamarii & 2 & 1.0 \\
Negative for Aspergillus species & 3 & 1.5 \\
\hline
\end{tabular}

Table 4. Aspergillus species isolated from the different varieties of peanuts.

\begin{tabular}{|c|c|c|c|c|c|c|}
\hline \multirow[b]{2}{*}{ District } & \multirow[b]{2}{*}{ Peanut variety } & \multicolumn{5}{|c|}{ Aspergillus species isolated } \\
\hline & & A. flavus L-strain & A. flavus S-strain & A. parasiticus & A. niger & A. tamarii \\
\hline \multirow[t]{5}{*}{ Busia } & Valencia red & $19(31.7 \%)$ & $7(23.3 \%)$ & $2(28.6 \%)$ & $0(0.0 \%)$ & $0(0.0 \%)$ \\
\hline & Uganda local red & $19(31.7 \%)$ & $9(30.0 \%)$ & $2(28.6 \%)$ & $0(0.0 \%)$ & $0(0.0 \%)$ \\
\hline & Homabay local & $20(33.3 \%)$ & $12(40.0 \%)$ & $1(14.2 \%)$ & $0(0.0 \%)$ & $0(0.0 \%)$ \\
\hline & Local red & $2(3.3 \%)$ & $2(6.7 \%)$ & $2(28.6 \%)$ & $2(100 \%)$ & $0(0.0 \%)$ \\
\hline & Total & $60(60.6 \%)$ & $30(30.3 \%)$ & $7(7.1 \%)$ & $2(2 \%)$ & $0(0)$ \\
\hline \multirow[t]{4}{*}{ Kisii central } & Valencia red & $12(54.6 \%)$ & $49(79.0 \%)$ & $4(33.3 \%)$ & $1(25.0 \%)$ & $0(0.0 \%)$ \\
\hline & Uganda local red & $5(22.7 \%)$ & $5(8.1 \%)$ & $3(25.0 \%)$ & $2(50.0 \%)$ & $1(50.0 \%)$ \\
\hline & Homabay local & $5(22.7 \%)$ & $8(12.9 \%)$ & $5(41.7 \%)$ & $1(25.0 \%)$ & $1(50.0 \%)$ \\
\hline & Total & $22(21.6 \%)$ & $62(60.8 \%)$ & $12(11.7 \%)$ & $4(3.9 \%)$ & $2(2 \%)$ \\
\hline
\end{tabular}

species was detected in Uganda local red and Homabay local peanut varieties at similar rates of $50 \%$ (Table 4 ).

The rate in percentage of each species was calculated based on the total number of isolates of each species in each district of study.

\section{Discussion}

\section{Occurrence of Aspergillus Species in Peanuts from Busia and Kisii Central Districts}

This study identified the Aspergillus species in peanuts from Busia and Kisii central that are involved in the production of aflatoxins. The predominant $A s$ pergillus species across the districts were A. flavus S-strain and Aspergillus flavus L-strain, with an incidence of $60.2 \%$ and $58.8 \%$ respectively. Aspergillus flavus particularly the $\mathrm{L}$ and $\mathrm{S}$ strain have been documented as the common species that grow and produce aflatoxins in foods including peanuts than other Aspergillus species [15]. These Aspergillus species have been isolated at slightly higher incidences in peanuts in a previous study [16], S-strain at $78 \%$ and L-strain at $68 \%$. The difference in incidences between the current and the previous studies could have been contributed by difference in sample sizes and the specific study 
districts.

Aspergillus flavus L-strain was the most common isolate (58.8\%) in peanut samples from Busia district while $A$. flavus $\mathrm{S}$-strain was the most common strain (60.2\%) in peanut isolates from Kisii central district. This might be contributed by difference in weather conditions between the two study districts. Aspergillus flavus $\mathrm{S}$-strain contains aflatoxin $\mathrm{Q}(\mathrm{aflQ})$ toxigenic genes which usually produce high aflatoxins in wet conditions while Aspergillus flavus L-strain contains aflatoxin $\mathrm{D}$ toxigenic genes that produce high aflatoxins in dry conditions [17]. The results are in line with other studies [18]. The high incidence of A. flavus S-strain particularly in Kisii central that produces aflatoxin [19] and in particular, the most potent Aflatoxin $B_{1}[19]$, indicates a risk of aflatoxin contamination of peanuts in areas in the western Kenya with wet climatic conditions which enhances the growth and production of aflatoxins mainly by Aspergillus flavus.

Aspergillus parasiticus, Aspergillus niger and Aspergillus tamarii were isolated in this study at overall mean occurrences of $9.3 \%, 2.9 \%$ and $1 \%$ respectively in peanuts from the two study districts. The low occurrences of these three Aspergillus species in the study areas is in line with the reports of Mutegi et al., (2010) [19], who documented these species in other districts of Kenya at comparable low occurrences. Aspergillus parasiticus was the third most common isolate at $12 \%$ and $6.9 \%$ in peanut samples for Kisii central district and Busia district respectively. Aspergillus parasiticus is known to be common in wet climatic conditions which facilitates growth and aflatoxin production especially aflatoxin G1 [19]. This could explain the high occurrence of Aspergillus parasiticus in Kisii central district compared to Busia. The results are consistent with previous study on peanuts by Mutegi et al. (2009) [20] where Aspergillus parasiticus was documented as the third most common Aspergillus species after A. flavus S-strain and Aspergillusflavus L-strain in the production of aflatoxin in peanut samples. The confirmation of occurrence of other species that produce toxins such as $A$. nigerand A. tamarii which also producecyclopiazonic acid [21], suggests that there is need to screen peanuts not just for aflatoxins but also for other carcinogenic mycotoxins.

Distribution of Aspergillus species in the different varieties of peanuts

The results of this study showed that all the varieties of peanuts sampled from both Busia and Kisii central districts were contaminated with at least one or more of A. flavus L-strain, A. flavus S-strain, A. parasiticus, Aspergillus niger and $A$. tamarii species. All the Aspergillus species were isolated in all the peanuts varieties from Busia district except $A$. niger which was detected in peanuts of Local variety while $A$. tamarii was not detected at all. Overall, Aspergillus flavus L-strain was the most highly detected strain $(60.6 \%)$ in all the peanut varieties collected from Busia district followed by Aspergillus flavus S-strain at $30.3 \%$ occurrence. These findings are similar to previous studies that reported Aspergillus flavus L-strain and Aspergillus flavus S-strain were the most common species involved in production of aflatoxins in foods including peanuts [17]. Other stu- 
dies also reported that Aspergillus flavus L-strain which contains aflD toxigenic genes produced more aflatoxin in dry weather conditions compared to Aspergillus flavus $\mathrm{S}$-strain [20].

Aspergillus flavus L-strain was found in peanuts of Homabay local variety at an occurrence of $33.3 \%$ while Aspergillus flavus S-strain had $40 \%$ in the same variety from Busia. This could have probably been as a result of high susceptibility of the local variety to crop diseases and pests, which result in plant stress thereby predisposing peanuts to the growth of Aspergillus flavus particularly the most toxigenic Aspergillus flavus $S$ strain [22]. Aspergillus parasiticus was also found to be contaminating all the peanut varieties from the study district but highly isolated in peanuts of Local red, Valencia red and Uganda local varieties at similar rates of $28.6 \%$. This could be due to the fact that Aspergillus parasiticus grows and produce aflatoxins even in improved peanuts varieties such as Valencia red. Aspergillus niger was only detected in peanuts of Local red variety from Busia. This indicates that this peanut variety from the area is more highly susceptible to the growth of Aspergillus including the less common species. The results are in line with a previous study [22] that documented that local peanuts varieties such as Local red, Homabay local and Uganda local red are more susceptible to diseases such as stem rot and mould which facilitates the growth of Aspergillus species.

In peanut varieties from Kisii central district, all the strains; Aspergillus flavus S-strain, Aspergillus flavus L-strain, Aspergillus parasiticus, A. niger except $A$. tamarii were isolated in all the varieties with higher occurrence (60.8\%) of $A s$ pergillus flavus $S$-strain. This is because the species grows better in wet weather conditions compared to other Aspergillus species resulting to its high occurrence [23]. This could have contributed to its high detection in peanuts of Valencia red variety (79\%). Aspergillus flavus L-strain had low occurrence (21.6\%) in all varieties from Kisii central. However, it's important to note that it was also detected highly in peanuts of Valencia red variety compared to other varieties. This suggests that the variety was more susceptible to Aspergillus species contamination than other varieties. This could probably be contributed by sowing of $A s$ pergillus contaminated Valencia red variety seeds from the supplier in the district which resulted to contaminated harvests.

Aspergillus parasiticus species had a higher incidence (41.7\%) in Homabay local variety compared to other varieties. Aspergillus niger had higher occurrence in peanuts of Uganda local red while Aspergillus tamarii was detected in peanuts of Uganda local red and Homabay local varieties at similar rates of 50\%. This could be probably due to higher susceptibility of local varieties to crop pests and diseases which facilitates Aspergillus species contamination including the less common species [24]. The result is in line with the study of Mutegi et al. (2009) [20] who showed that peanuts of local varieties have a higher likelihood of being contaminated with aflatoxin than improved varieties. Previous studies have documented higher susceptibility of local peanut varieties to improved va- 
rieties in fungal contamination including Aspergillus species in the United Kingdom [25].

\section{Conclusion}

Aspergillus flavus L-strain was the most common isolate in peanuts from the dry district of Busia while $A$. flavus S-strain was the most prevalent strain in peanuts from the wet district of Kisii central. Overall, the occurrence of Aspergillus flavus L-strain and $A$. flavus $\mathrm{S}$-strain were significantly higher than other species identified $(\mathrm{H}=15.55, \mathrm{df}=4, \mathrm{P}=0.004)$ in peanuts from the two districts. However, A. flavus $\mathrm{S}$-strain was the most detected species $(\mathrm{F}=3.15, \mathrm{df}=25, \mathrm{P}=$ $0.031)$.

\section{Acknowledgements}

We would like to thank Kenyatta University Ethics Review Committee for the approval of this study. We also acknowledge the National Commission for Science, Technology and Innovation (NACOSTI), Kenya for issuing a Research permit for the study. We are grateful to all the peanuts farmers for their cooperation during sample collection. Finally, we are also grateful to George K. Gathumbi of Bora Limited Laboratory, Kenya for his contribution in this research work.

\section{Conflicts of Interest}

The authors declare no conflicts of interest regarding the publication of this paper.

\section{References}

[1] Khlangwiset, P., Shepherd, G. and Wu, F. (2011) Aflatoxins and Growth Impairment: Critical Reviews in Toxicolology, 118, 817-824.

[2] Liu, T. and Wu, F. (2010) Global Burden of Aflatoxin Induced Hepatocellur Carcinoma. A Risk Assess. Environmental Health Perspectives, 118, 818-824. https://doi.org/10.1289/ehp.0901388

[3] Fung, F. (2004) Health Effects of Mycotoxins: A Toxicological Overview. Journal of Clinical Toxicology, 42, 217-234.

[4] WHO (2008) World Health Statistics. WHO Press, Geneva, 1-5.

[5] Lewis, L., Onsongo, M., Njapau, H., Schur-Rogers, H., Laber, G., Kieszak, S., et al. (2005) Aflatoxin Contamination of Commercial Maize Products during an Outbreak of Acute Aflatoxicosis in Eastern and Central Kenya. Environmental Health Perspective, 113, 1763-1767. https://doi.org/10.1289/ehp.7998

[6] Mutegi, C., Wagacha, M., Kimani, J., Otieno, G., Wanyama, R., Hell, K., et al. (2013) Incidence of Aflatoxin in Peanuts from Markets in Western, Nyanza and Nairobi Provinces of Kenya and Related Market Traits. Journal of Stored Produce Research, 52, 118-127. https://doi.org/10.1016/j.jspr.2012.10.002

[7] Ogwang, T. (2006) Status of Groundnut Production in Busia Districts: Lucrative legume Project. Stakeholders Meeting, Milimani Resort, Kisumu. 
[8] Kipkoech, A., Okiror, M., Okalebo, J. and Maritim, H. (2007) Production Efficiency and Economic Potential of Different Soil Fertility Management Strategies among Groundnut Farmers of Kenya. Science World Journal, 2, 51714-77951.

[9] Whitaker, T. (2006) Sampling Foods for Mycotoxin. Food Addictives and Contaminants, 23, 50-61. https://doi.org/10.1080/02652030500241587

[10] Probst, C., Njapau, H. and Cotty, P. (2007) Outbreak of an Acute Aflatoxicosis in Kenya. Identification of the Causal Agent. Applied and Environmental Microbiology, 73, 2762-2764.

[11] Klich, M. (2002) Identification of Common Aspergillus Species. Mycoscience, 48, 71-80. https://doi.org/10.1007/S10267-006-0336-2

[12] Mellon, J. and Cotty, P. (2004) Expression of Pectinase Activity among Aspergillus flavus Isolates from Southwestern United States. Mycopathology, 157, 333-338. https://doi.org/10.1023/B:MYCO.0000024181.36900.15

[13] Kornerup, A. and Wanscher, J. (1978) Metheun Hand Book of Colour. 3rd Edition, Metheun London Ltd., London, 144-148.

[14] Cotty, P. and Cardwell, K. (1999) Divergence of West African and North American Communities of Aspergillus section Flavi. Applied and Environmental Microbiology, 65, 2264-2266.

[15] Mutungi, C., Lamuka, P., Arimi, S., Gathumbi, J. and Onyango, C. (2008) The Fate of Aflatoxins during Processing of Maize into Muthokoi: A Traditional Kenyan Food. Food Controlling, 19, 714-721. https://doi.org/10.1016/j.foodcont.2007.07.011

[16] Mutegi, C., Hendriks, S. and Ngugi, H. (2010) The Extent of Aflatoxin and Aspergillus Section Flavi, Penicillium Spp. and Rhizopus spp. Contamination of Peanuts from Households in Western Kenya and the Causative Factors of Contamination. Research Space, 1-107.

[17] Gachomo, E., Mutitu, E. and Kotchoni, O. (2004) Diversity of Fungal Species Associated with Peanuts in Storage and the Levels of Aflatoxins in Infected Samples. International Journal of Agriculture and Biology, 6, 955-959.

[18] Udoh, J., Cardwell, K. and Ikotun, T. (2000) Storage Structures and Aflatoxin Content of Maize in Five Agro-Ecological Zones of Nigeria. Journal of Stored Products Research, 36, 187-201. https://doi.org/10.1016/S0022-474X(99)00042-9

[19] Ehrlich, K., Kobbeman, K., Montalbano, B. and Colty, P. (2007) Aflatoxin Producing Aspergillus Species from Thailand. International Journal of Food Microbiology, 114, 153-159. https://doi.org/10.1016/j.ijfoodmicro.2006.08.007

[20] Mutegi, C., Ngugi, H., Hendriks, S. and Jones, R. (2009) Prevalence and Factors Associated with Aflatoxin Contamination of Peanuts from Western Kenya. International Journal of Food Microbiology, 130, 27-34. https://doi.org/10.1016/j.ijfoodmicro.2008.12.030

[21] Bayman, P., Baker, J., Doster, M., Michaildes, T. and Mahoney, N. (2002) Ochratoxin Production by the Aspergillus ochraceus Group and Aspergillus alliaceus. Applied and Environmental Microbiology, 68, 2326-2329. https://doi.org/10.1128/AEM.68.5.2326-2329.2002

[22] Chapin, J., Dorner, J. and Thomas, J. (2004) Association of a Burrower Bug (Heteroptera cydnidae) with Aflatoxin Contamination of Peanut Kernels. Journal of Entomology Science, 39, 71-83. https://doi.org/10.18474/0749-8004-39.1.71

[23] Kasno, A. (2004) Prevention of Aspergillus flavus Infection and Aflatoxin Contamination in Groundnut. Journal of Penelitian dan Pengembangan Pertanians, 23, 
75-81.

[24] Robertson-Hoyt, L., Payne, G., Isakeit, T., Maragos, C., Molnar, T. and Holland, J. (2007) Relationships among Resistances to Fusarium and Aspergillus Ear Rots and Contamination by Fumonisin and Aflatoxin in Maize. Phytopathology, 99, 311-317. https://doi.org/10.1094/PHYTO-97-3-0311

[25] Middleton, K., Pande, S., Sharma, S. and Smith, D. (1994) Diseases in Groundnut Crop: A Scientific Basis for Improvement. Chapman and Hall Journal, 336-378. 